often only one is desired. Researchers have developed a catalyst that changes its configuration when exposed to light and that can generate one enantiomer or the other, depending on the position of the catalyst's rotating parts.

The catalyst is based on a molecular 'motor' comprising two parts rotating around a fixed axle. The motor moves through a four-step cycle in which each change in position is triggered by light. At each position, the relative orientation of the two moving parts controls the spatial position of the reactant in a chemical reaction, producing either one or the other enantiomer, or a mix of the two.

Using just one molecule of the catalyst, Jiaobing Wang and Ben Feringa at the University of Groningen in the Netherlands produced both enantiomers in a sequential manner.

Science doi:10.1126/

science.1199844 (2011)

\section{IMMUNOLOGY}

\section{Modulating malaria's mayhem}

The malaria parasite wreaks havoc in the body, in part by activating receptors that normally detect the presence of microbes and initiate innate immune responses. By interfering with these 'Tolllike' receptors in a mouse model of cerebral malaria, the worst symptoms of the disease can be prevented, say Ricardo Gazzinelli at the University of Massachusetts Medical School in Worcester and his team.

By activating Toll-like receptors, the malaria parasite triggers the excessive release of inflammatory immune molecules called cytokines. The researchers used a small molecule to block Toll-likereceptor activation in mice after they had been infected with malaria. The inhibitor vastly improved the animals' survival and decreased the prevalence of cerebral malaria symptoms such as seizures, even though it did not decrease the number of

parasites. The strategy might help to cut malaria mortality in the absence of a preventive vaccine, the authors say.

Proc. Natl Acad. Sci. USA

doi:10.1073/pnas.1015406108 (2011)

\section{HYDROLOGY}

\section{Melting to} the max

As the climate warms, a larger proportion of precipitation is likely to take the form of rain and less as snow. The snow that does still accumulate on mountains during winter will subsequently melt faster in the spring, increasing peak discharge and flood risk.

Amilcare Porporato and his colleagues at Duke University in Durham, North Carolina, used a model to calculate how seasonal temperature affects snow accumulation, melting and mountain run-off. In the model, peak discharge increases to a maximum in a warming environment despite reduced snowpack, and then drops off as less and less snow accumulates in winter.

In the real world, climatechange-driven alterations in the timing and volume of mountain snowmelt could increase the risk of both flooding and summer droughts in the catchment areas of meltwater-fed rivers. Geophys. Res. Lett. doi:10.1029/ 2010GL046477 (2011)

\section{MOLECULAR BIOLOGY}

\section{When DNA repair goes wrong}

Mistakes in the DNA sequence are constantly being repaired, but some repair mechanisms are themselves error-prone. One, called break-induced replication (BIR), is initiated at sites at which both DNA strands are broken, and mimics the process of DNA replication. However, its rate of frameshift mutation - a type of mutation that almost always results in loss of gene function - is up to 2,800 times higher than that of normal replication.

COMMUNITY CHOICE

The most viewed papers in science

\title{
Tobacco smoke, take three
}

\section{- HIGHLY READ \\ on pubs.acs.org in January 2011}

The dangers of second-hand cigarette smoke are well known, but nicotine can linger on indoor surfaces and generate 'third-hand' smoke, either through its re-emission into the air or by direct ingestion from surfaces. Researchers have found that nicotine on surfaces can produce aerosols of potentially toxic oxidation products, by reacting with certain oxidizing gases when exposed to conditions typical of those found in the home.

Yael Dubowski and her team at the Israel Institute of Technology in Haifa doped cellulose powder, paper and cotton samples with a nicotine suspension and then exposed them to ozone and nitrogen oxide gases. Aerosols were collected and analysed.

The authors found that relative humidity and the type of surface affected the number and size distribution of the aerosol particles. Aerosol formation was greatest on cellulose in dry air, whereas much fewer aerosols formed from paper. Environ. Sci. Technol. 45, 328-333 (2011)

Anna Malkova at Indiana University-Purdue University Indianapolis and her team measured mutation rates during BIR in yeast. They found that errors occurred not just close to the site of the double-stranded break, but also farther down the DNA. Most of the mistakes were generated by DNA polymerase enzymes, which replicate DNA and are generally thought to be accurate. The authors say that one of the factors leading to the high mutation rate was elevated levels of nucleotides and that BIR could be an important source of the mutations that drive cancer and evolution. PLoS Biol. 9, e1000594 (2011)

\section{PALAEOANTHROPOLOGY}

\section{No marathons for Neanderthals}

Fossilized heel bones suggest that Neanderthals were not built to run long distances.

By observing contemporary human distance runners on a treadmill, David Raichlen at the University of Arizona in Tucson and his team found that athletes with shorter heel

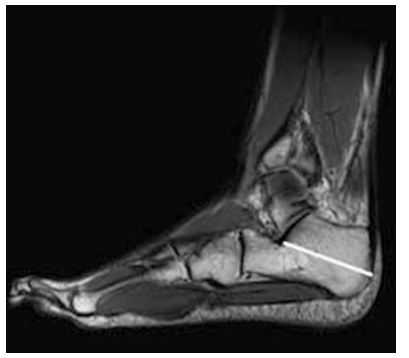

bones, or calcanei (pictured, white line), tended to run more economically - consuming less oxygen to maintain the same speed - than those with longer ones. Calcanei from 7 Neanderthals were, on average, longer than the same bone from 13 ancient humans and the 8 treadmill runners.

Our African-dwelling ancient ancestors may have evolved feet adapted to chasing animals to the point of heat exhaustion. Neanderthals, who lived in colder, forested European and Asian habitats, may instead have attacked prey at close range, the authors say. J. Hum. Evol. 60, 299-308 (2011)

\section{ONATURE.COM}

For the latest research published by Naturevisit:

www.nature.com/latestresearch 